\title{
Neuromodulation, Specialized Proresolving Mediators, and Resolution of Pain
}

\author{
Xueshu Tao ${ }^{1} \cdot$ Michael S. Lee ${ }^{1} \cdot$ Christopher R. Donnelly ${ }^{1} \cdot$ Ru-Rong $\mathrm{Ji}^{1,2,3}$ (I) \\ Published online: 21 July 2020 \\ (C) The American Society for Experimental NeuroTherapeutics, Inc. 2020
}

\begin{abstract}
The current crises in opioid abuse and chronic pain call for the development of nonopioid and nonpharmacological therapeutics for pain relief. Neuromodulation-based approaches, such as spinal cord stimulation, dorsal root ganglion simulation, and nerve stimulation including vagus nerve stimulation, have shown efficacy in achieving pain control in preclinical and clinical studies. However, the mechanisms by which neuromodulation alleviates pain are not fully understood. Accumulating evidence suggests that neuromodulation regulates inflammation and neuroinflammation - a localized inflammation in peripheral nerves, dorsal root ganglia/trigeminal ganglia, and spinal cord/brain - through neuro-immune interactions. Specialized proresolving mediators (SPMs) such as resolvins, protectins, maresins, and lipoxins are lipid molecules produced during the resolution phase of inflammation and exhibit multiple beneficial effects in resolving inflammation in various animal models. Recent studies suggest that SPMs inhibit inflammatory pain, postoperative pain, neuropathic pain, and cancer pain in rodent models via immune, glial, and neuronal modulations. It is noteworthy that sham surgery is sufficient to elevate resolvin levels and may serve as a model of resolution. Interestingly, it has been shown that the vagus nerve produces SPMs and vagus nerve stimulation (VNS) induces SPM production in vitro. In this review, we discuss how neuromodulation such as VNS controls pain via immunomodulation and neuro-immune interactions and highlight possible involvement of SPMs. In particular, we demonstrate that VNS via auricular electroacupuncture effectively attenuates chemotherapy-induced neuropathic pain. Furthermore, auricular stimulation is able to increase resolvin levels in mice. Thus, we propose that neuromodulation may control pain and inflammation/neuroinflammatioin via SPMs. Finally, we discuss key questions that remain unanswered in our understanding of how neuromodulation-based therapies provide short-term and long-term pain relief.
\end{abstract}

Key Words Auricular stimulation · chemotherapy $\cdot$ dorsal root ganglion $\cdot$ fish oil $\cdot$ resolvin $\cdot$ vagus nerve stimulation

\section{Abbreviations \\ aEA Auricular electroacupuncture \\ AT-RvD1 Aspirin-triggered resolvin D1 \\ CRG Carrageenan \\ CCI Chronic constriction injury \\ CFA Complete Freund's adjuvant}

Ru-Rong Ji

ru-rong.ji@duke.edu

1 Center for Translational Pain Medicine, Department of Anesthesiology, Duke University Medical Center, Durham, NC 27710, USA

2 Department of Neurobiology, Duke University Medical Center, Durham, NC 27710, USA

3 Department of Cell Biology, Duke University Medical Center, Durham, NC 27710, USA

$\begin{array}{ll}\text { CGRP } & \text { Calcitonin gene-related peptide } \\ \text { CIPN } & \text { Chemotherapy-induced peripheral } \\ & \text { neuropathy } \\ \text { CNS } & \text { Central nervous system } \\ \text { COX } & \text { Cyclooxygenase } \\ \text { DHA } & \text { Docosahexaenoic acid } \\ \text { DRG } & \text { Dorsal root ganglion } \\ \text { EA } & \text { Electroacupuncture } \\ \text { EPA } & \text { Eicosapentaenoic acid } \\ \text { GPCR } & \text { G protein-coupled receptors } \\ \text { IFM } & \text { Inflammatory mediator } \\ \text { JNK } & \text { c-Jun N-terminal kinase } \\ \text { LOX } & \text { Lipoxygenase } \\ \text { MAPK } & \text { Mitogen-activated protein kinase } \\ \text { NPD1 } & \text { Neuroprotectin D1 } \\ \text { PK } & \text { Pharmacokinetics } \\ \text { PNS } & \text { Peripheral nervous system }\end{array}$




$\begin{array}{ll}\text { PTX } & \text { Paclitaxel } \\ \text { PUFA } & \text { Polyunsaturated fatty acids } \\ \text { RvD1 } & \text { Resolvin D1 } \\ \text { RvE1 } & \text { Resolvin E1 } \\ \text { SPM } & \text { Specialized proresolving mediator } \\ \text { TENS } & \text { Transcutaneous nerve stimulation } \\ \text { TMS } & \text { Transcranial magnetic stimulation } \\ \text { TRPA1 } & \text { Transient receptor potential ion channel } \\ & \text { subtype A1 } \\ \text { TRPV1 } & \text { Transient receptor potential ion channel } \\ & \text { subtype V1 } \\ \text { VNS } & \text { Vagus nerve stimulation }\end{array}$

\section{Inflammation and Pain}

Inflammation has five main cardinal symptoms: rubor (redness), tumor (swelling), calor (increased heat), dolor (pain), and functio laesa (loss of function). Pain is initially sensed by physical and chemical activation of nociceptive sensory neurons (nociceptors) [1]. Nociceptors, finely tuned to sense the presence of potentially hazardous stimuli, can be directly activated by inflammatory mediators (IFMs) through their receptors expressed on nociceptors [2,3]. These IFMs include small peptides (e.g., calcitonin gene-related peptide (CGRP) and substance $\mathrm{P}$, bradykinin), lipids (e.g., prostaglandins and epoxyeicosatrienoic acids), proinflammatory cytokines and chemokines (e.g., IL-1 $\beta$, TNF, CCL2), ATP, and microRNAs [4-9]. Physiological pain mediated by nociceptor activation is normally protective, sending a warning signal to the brain to trigger avoidance of a given noxious stimulus. Recent evidence suggests that pain also protects against infection through nociceptor-mediated release of CGRP, which can contribute to the regulation of host immunity $[10,11]$. However, due to sensitization of peripheral nociceptors (peripheral sensitization), pain can become a pathological phenomenon $[12,13]$. Other types of pain such as headaches and migraines may also originate from central sensitization within the central nervous system (CNS) $[14,15]$.

After tissue injury and inflammation, IFMs induce peripheral sensitization via modulation of ion channels involved in nociceptive neurotransmission, including voltage-gated sodium channels (Nav1.7, Nav1.8, Nav1.9) and TRP channels (TRPA1 and TRPV1), mediated chiefly through protein kinase pathways $[2,5]$. These ion channels are highly enriched in nociceptors [16, 17], and their expression and activities in nociceptors are regulated by $\mathrm{p} 38$ MAP kinase (MAPK) [18-21], in response to IFMs. Central sensitization drives widespread chronic pain $[14,15]$ and is regulated by NMDA receptors and extracellular signal-regulated kinase (ERK/ MAPK) in nociceptive neurons in the CNS [22, 23]. Mounting evidence suggests that loss of inhibitory synaptic transmission (disinhibition) is also a critical contributor to central sensitization following nerve injury-induced neuropathic pain [24-26]. While peripheral sensitization typically precedes central sensitization, central sensitization can be directly elicited after spinal cord injury (SCI) and traumatic brain injury (TBI), sending electrical and chemical signals back to sustain peripheral sensitization $[15,27]$.

It is now well understood that neuroinflammation is a critical driver in the pathogenesis of chronic pain [2]. Neuroinflammation is a local inflammation residing in neural tissues that can be characterized by peripheral immune cell infiltration and activation of glial cells, such as microglia and astrocytes in the CNS, as well as activation of satellite glial cells and Schwann cells in the PNS $[2,28]$. Painful insults such as nerve injury, joint injury, cancer, and chronic opioid treatment have been shown to produce reactive changes in glial cells [29]. Following activation, glia produce many powerful neuromodulators, such as cytokines and chemokines (TNF, IL-1 $\beta$, CCL2, CXCL1), via activation of MAP kinase signaling pathways. These glial messengers potentiate excitatory synaptic transmission and drive long-term potentiation in the spinal cord pain circuit via intracellular signaling, leading to enhanced pain states [29-32]. Notably, glial mediators, such as IL-1 $\beta$, IL-17, and brain-derived neurotrophic factor (BDNF) also suppress inhibitory synaptic transmission to drive central sensitization in pathological pain [33-35]. Additionally, neurogenic inflammation is another type of inflammation induced by nociceptor-mediated release of CGRP and substance $\mathrm{P}$ (SP), which contributes to inflammatory pain, migraine, and complex regional pain syndrome [36-38].

A primary function of inflammation is to eliminate the initial cause of cell injury, allowing for tissue repair and homeostasis. However, excessive and chronic inflammation is maladaptive and detrimental, emerging as a leading cause of many inflammatory and neurological diseases [2]. A major sign of progress in inflammation research is the realization that resolution of acute inflammation is an active biochemical process representing a new therapeutic frontier [39, 40]. In particular, resolution of acute inflammation requires the production of lipid-derived specialized proresolving mediators (SPMs), which is generated during the resolution phase and contributes to the resolution process [41, 42]. SPMs belong to a rapidly expanding family of molecules, including resolvins, protectins, and maresins, biosynthesized from omega- 3 unsaturated fatty acids docosahexaenoic acid (DHA) and eicosapentaenoic acids (EPA), two major components of fish oil dietary supplements [43] although some SPMs such as lipoxins are also biosynthesized from arachidonic acids [41] (Fig. 1). SPMs have shown to exert potent protective effects in animal models of segregated human diseases, such as arthritis, kidney injury, infection, sepsis, Alzheimer's, and cancer [44-52]. Additionally, an increasing body of evidence also points to a role of SPMs in promoting the resolution of pathological pain [52] (Table 1). 
a
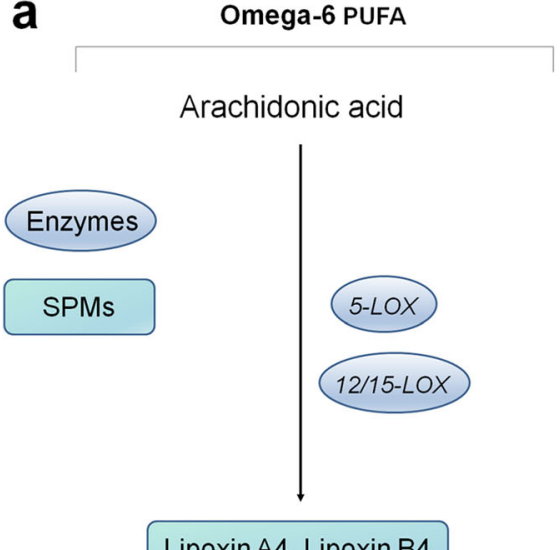

Lipoxin A4, Lipoxin B4

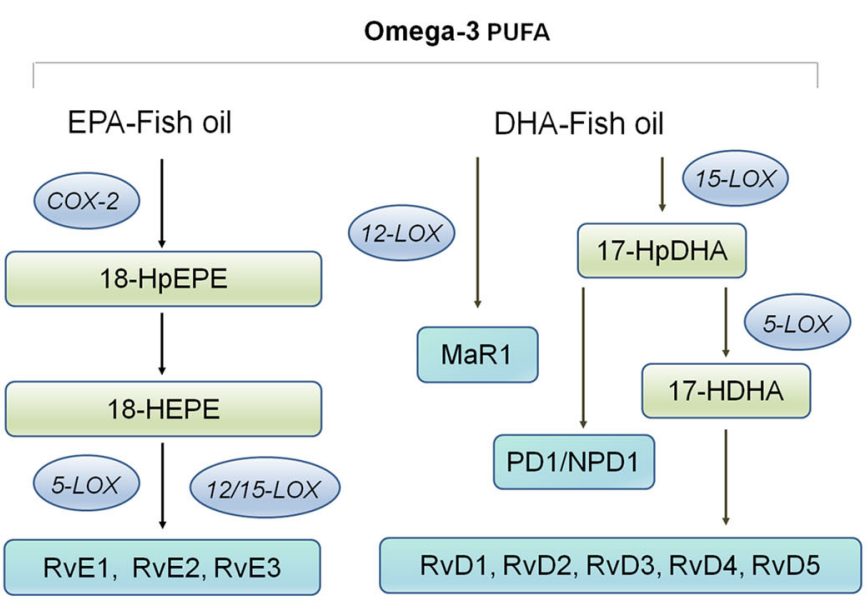

b

SPM and SPM receptors (GPCRs)

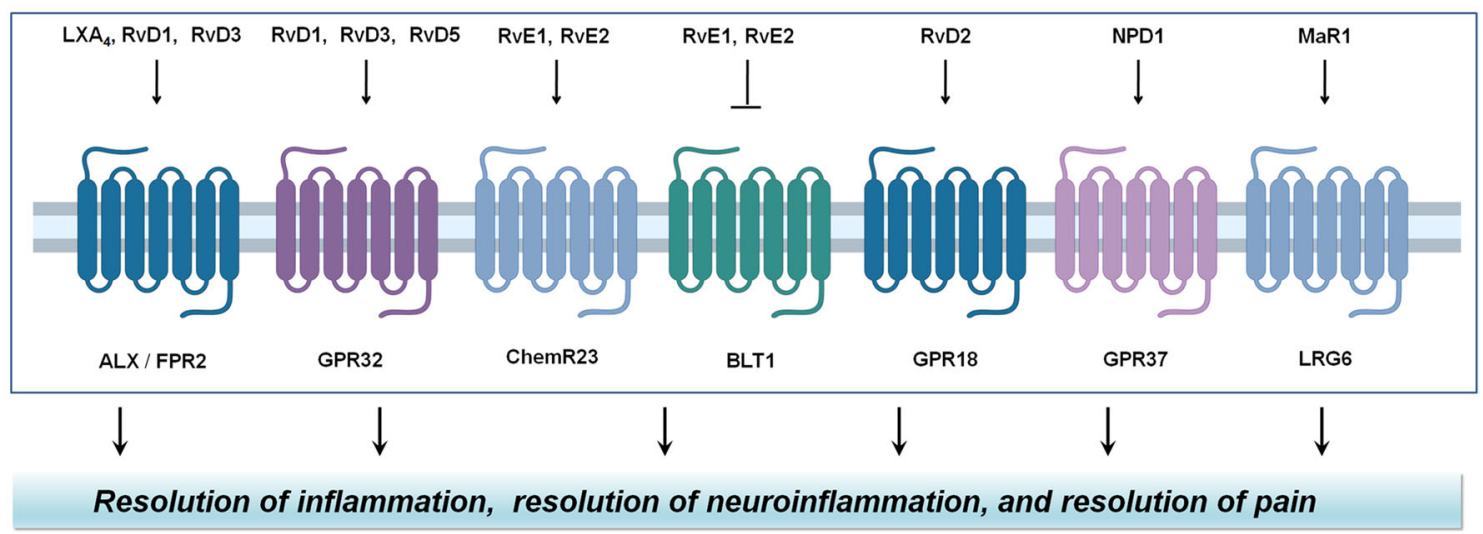

C

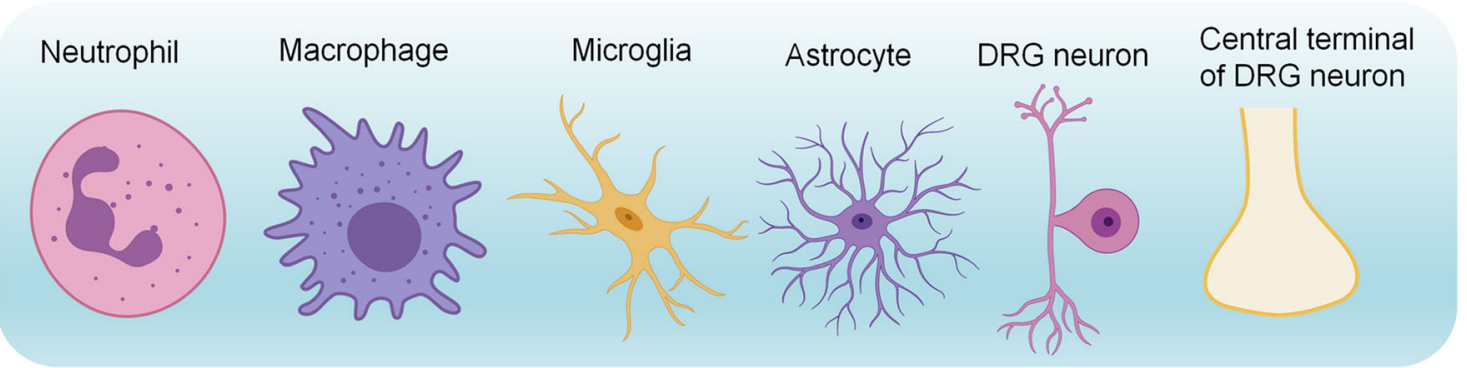

Fig. 1 SPMs control pathological pain via neuronal, glial, and immune regulations. (a) SPMs (e.g., resolvins, protectins, and maresins) are primarily biosynthesized from omega-3 polyunsaturated fatty acids EPA and DHA via LOX and COX enzymes. (b) SPMs activate their respective receptors (GPCRs) to regulate inflammation,

\section{SPMs Are Potent Pain Inhibitors in Different Pathological Conditions}

\section{Resolvins}

Resolvin D-series (e.g., RvD1-RvD5) and resolvin E-series (RvE1-RvE5) are derived from DHA and EPA, respectively. A growing body of evidence suggests that resolvins potently neuroinflammation, and neuronal and synaptic plasticity, leading to the resolution of pain. (c) SPM receptors are expressed on immune cells (neutrophils, macrophages), glial cells (microglia and astrocytes), and neurons (DRG neurons and their central terminals). Thus, SPMs promote pain resolution via immune, glial, and neuronal regulations reduce inflammatory pain. Intraplantar pretreatment of mice with very low doses (20 ng) of RvE1 and RvD1, given 10 min prior to tissue injury, is sufficient to prevent carrageenan (CRG)-induced inflammatory pain. RvE1 also reduces CRG-induced neutrophil infiltration and paw edema, along with decreased expression of proinflammatory cytokines and chemokines (e.g., TNF- $\alpha$, IL-1 $\beta$, IL-6, and CCL2) in inflamed paw tissues [53]. Intraplantar RvD1 or RvE1 resulted 
Table 1 SPMs reduce pathological pain in different rodent models

\begin{tabular}{|c|c|c|c|c|}
\hline Pain Model & SPMs & Species & Effects & References \\
\hline \multicolumn{5}{|c|}{ Acute inflammation pain } \\
\hline Formalin & RvD1, RvD2, RvE1, NPD1 & Mice & Spontaneous pain $\downarrow$ & {$[53-56]$} \\
\hline Capsaicin & RvD2, RvE1, NPD1, MaR1 & Mice & Spontaneous pain $\downarrow$ & {$[54-58]$} \\
\hline CRG & RvD1, RvE1 & Mice & $\begin{array}{l}\text { Heat hyperalgesia } \downarrow \\
\text { Mechanical allodynia } \downarrow\end{array}$ & {$[53]$} \\
\hline \multicolumn{5}{|c|}{ Chronic inflammation pain } \\
\hline CFA & RvD1, RvD2, RvE1, NPD1 & Mice & Heat hyperalgesia $\downarrow$ & {$[53,55,56]$} \\
\hline Arthritic pain & AT-RvD1 & Rats & Mechanical hyperalgesia $\downarrow$ & {$[57]$} \\
\hline \multicolumn{5}{|l|}{ Neuropathic pain } \\
\hline $\mathrm{CCI}$ & RvE1, NPD1 & Mice & Mechanical allodynia $\downarrow$ & {$[58,59]$} \\
\hline Spinal cord injury & LXA4 & Mice & Mechanical allodynia $\downarrow$ & {$[60]$} \\
\hline Chemotherapy & RvD1, RvD2, RvD5, MaR1 & Mice & Mechanical allodynia $\downarrow$ & {$[61]$} \\
\hline Postoperative pain & RvD1, RvD2, MaR1 & Rats, mice & Mechanical hyperalgesia and allodynia $\downarrow$ & {$[62,63]$} \\
\hline Cancer pain & RvD1, RvD2 & Mice & $\begin{array}{l}\text { Thermal hyperalgesia } \downarrow \\
\text { Mechanical allodynia } \downarrow\end{array}$ & {$[64]$} \\
\hline
\end{tabular}

in suppression of formalin-induced acute spontaneous pain [53]. The potent analgesic actions of resolvins in inflammatory pain are only partially related to its direct anti-inflammatory actions, as RvE1 is more effective in reducing inflammatory pain than reducing leukocyte trafficking in terms magnitude change [53]. Notably, RvD1, RvD1, and RvD2 were shown to differentially regulate the function of TRP channels, key regulators of inflammatory pain [65]. For example, intraplantar administration of RvE1 in mice abolished the spontaneous pain induced by capsaicin (a TRPV1 agonist) but not by mustard oil (a TRPA1 agonist). In contrast, intraplantar administration of RvD1 blocked TRPA1 but not TRPV1 agonistelicited pain [54]. Intraplantar post-treatment of RvD1 also reduced CFA-induced mechanical hyperalgesia and allodynia via TRPA1 inhibition [54]. Further, repeated systemic administration of AT-RvD1 effectively attenuated CFA-evoked mechanical hyperalgesia in rats [57] (Table 1). Resolvins have also been shown to reduce inflammatory pain via central actions. Intrathecal (spinal administration) post-treatment with either RvE1 and RvD1 rapidly reduced CFA-induced heat and mechanical hypersensitivity [57]. Additionally, pretreatment of RvE1 intrathecally, at very low doses ( 0.3 and $1 \mathrm{ng})$, $10 \mathrm{~min}$ prior to formalin injection, reduced formalin-induced 2nd phase pain, which is known to be mediated via central sensitization in the spinal cord [53]. Strikingly, the dose of RvE1 required to reduce the 2 nd phase pain was 100 times lower than that of morphine and 10,000 times lower than that of the COX-2 inhibitor NS-398 (Fig. 2a), underscoring the potency of resolvins [53].

$\mathrm{RvD} 2$ is a highly potent inhibitor of TRPV1 $\left(\mathrm{IC}_{50}=0.1 \mathrm{nM}\right)$ and TRPA1 $\left(\mathrm{IC}_{50}=2 \mathrm{nM}\right)$ in primary sensory neurons, whereas RvE1 and RvD1 selectively inhibit TRPV1 and TRPA1, respectively [55]. Accordingly, RvD2, RvE1, and RvD1 differentially regulated TRPV1- and TRPA1-mediated acute inflammatory pain and spinal cord synaptic plasticity. Furthermore, intrathecal RvD2 was found to reverse CFA-induced inflammatory pain without altering motor function. Intrathecal RvD2 also reversed C-fiber stimulation-evoked long-term potentiation in the spinal cord. Collectively, these findings suggest distinct roles of resolvins in regulating TRP channels and highlight the role of RvD2 as a potent endogenous inhibitor for TRPV1/TRPA1 and inflammatory pain [55].

Resolvins have also been shown to exhibit potent analgesic actions in rodent models of postoperative pain (Table 1). Postoperative pain after prolonged muscle retraction lasts 3 4 weeks in humans and rodents [68]. RvE1 and RvD1 were found to prevent this paw incision-induced postoperative pain in mice and rats [62]. In a skin-muscle retraction model, surgery-induced mechanical hypersensitivity can last up to 4 weeks, but was prevented by a single RvD1 treatment in rats [62]. Similarly, clinical thoracotomy is associated with high incidence of chronic pain, but intrathecal resolvins were interestingly shown to slow and eventually prevent the development of chronic, post-thoracotomy pain [63]. Notably, post-treatment of RvD1 at later time points (e.g., postoperative day 9) produced only transient pain relief ( $<1$ day) [62], highlighting a timedependent role of resolvin in this model. In a mouse model of postoperative pain induced by tibial bone fracture, intrathecal post-treatment of RvD1 and RvD5, but not RvD3 and RvD4, also effectively reduced mechanical and cold allodynia [67].

Several SPMs have also shown efficacy in reducing neuropathic pain after nerve trauma and chemotherapy [59, 61]. Intrathecal injection RvE1 reduced CCI (chronic constriction injury of the sciatic nerve)-induced neuropathic pain and microglial reaction in the spinal cord [59]. Chemotherapyinduced peripheral neuropathy (CIPN) is a dose-limiting side 
a

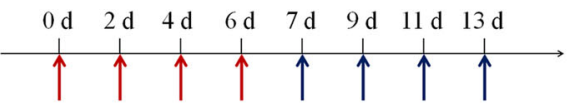

b

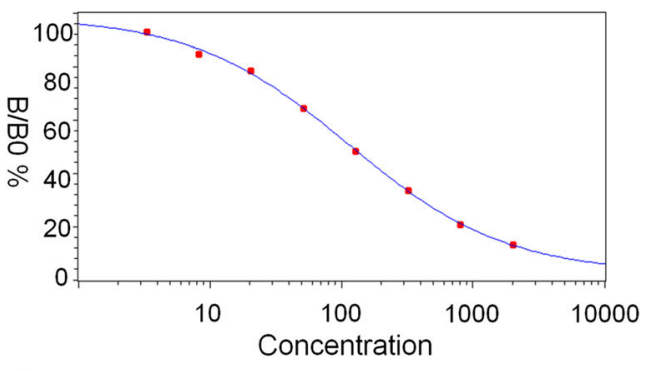

C

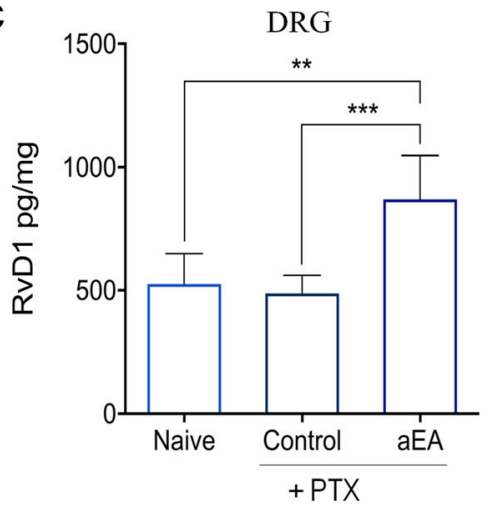

d

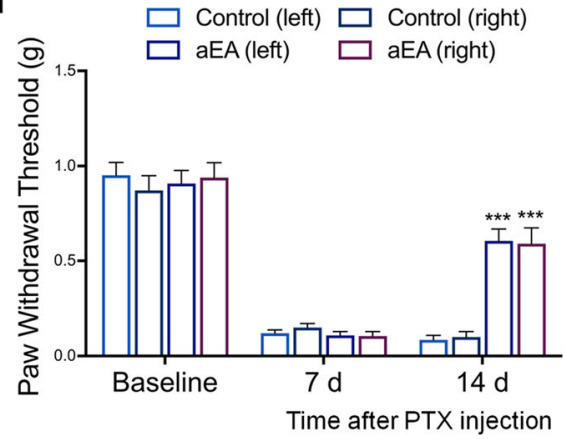

effect of many commonly utilized cytotoxic chemotherapy drugs used to treat cancer, such as paclitaxel $[69,70]$. CIPNassociated neuropathic pain often results in reduced quality of life, as well as dose reductions or discontinuation of cancer therapy [71, 72]. Given a lack of FDA-approved treatment for CIPN, development of a novel, safe treatment for neuropathic pain after chemotherapy remains a pressing issue. To this end, we recently evaluated the analgesic actions of D-series resolvins in paclitaxel-induced CIPN. Notably, intrathecal injection of RvD1 and RvD2 (100 ng, i.t.) at 2 weeks led to reversal of paclitaxel-induced mechanical allodynia in mice [61].

Beyond alleviating neuropathic pain caused by cancer treatments like chemotherapy, RvD2 itself has also exhibited
Fig. 2 Auricular vagus nerve stimulation via EA (aEA) increases RvD1 in DRG tissue and reduces neuropathic pain after paclitaxel (PTX)-induced CIPN in mice. (a) Experimental paradigm for PTX treatment and unilateral aEA. (b) Standard curve of RvD1 ELISA. $R=0.999$. (c) aEA increases RvD1 levels in DRG 14 days after PTX treatment. $* * P<0.01$, $* * * P<0.001$, one-way ANOVA with Bonferroni's post hoc test, $n=6$ mice per group. (d) aEA reduces mechanical allodynia 14 days after PTX treatment. $* * * P<0.001$ versus the control group without aEA. Two-way ANOVA with Bonferroni's post hoc test, $n=10$ mice per group. Methods for Fig. 2: Adult CD1 mice of both sexes (25-35 g) were purchased from Charles River Laboratories. All animals were maintained at the Duke University Animal Facility. Animal experiments were approved by the Animal Care Committee of Duke University. The location of auricular EA (aEA) is similar to human points given through two acupuncture needles on the core area of the right ear. The parameters of EA are $1 \mathrm{~mA}, 15 \mathrm{~Hz}, 0.1 \mathrm{~ms}$ pulse, and $30 \mathrm{~min}$. To minimize stress during aEA, mice were lightly anesthetized mice with $1 \%$ isoflurane. aEA was given every other day for 1 week on days $7,9,11$, and 13 . To induce CIPN in mice, 4 intraperitoneal injections of paclitaxel $(2 \mathrm{mg} / \mathrm{kg}$ per injection, Sigma) were given on days $0,2,4$, and 6 . For testing the mechanical pain threshold, the plantar surface of each hindpaw was stimulated with a series of von Frey fibers with logarithmically incrementing stiffness (0.02-2.56 g, Stoelting), presented perpendicular to the plantar surface. The $50 \%$ paw withdrawal threshold was calculated using Dixon's up-down method [66]. All data were expressed as the mean \pm SEM. All data were analyzed by one-way or repeated measures two-way ANOVA, followed by Bonferroni's post hoc test. $P<0.05$ was taken as statistically significant. Mouse RvD1 ELISA was conducted as previously described [67]. ELISA kit was purchased from Cayman Chemicals (Catalog number, 500380). The detection sensitivity of this ELISA kit is $15 \mathrm{pg} / \mathrm{ml}$, which is sufficient to detect RvD1 levels in DRG samples. Lumbar DRG tissues from both sides were collected from naïve animals and animals 14 days after PTX treatment. DRGs were homogenized in a lysis buffer containing protease and phosphatase inhibitors. Tissue samples were centrifuged at $12,500 \times \mathrm{g}$ for $10 \mathrm{~min}$ and the supernatant was collected. Protein concentrations were determined by BCA Protein Assay (Pierce). For each reaction in a 96-well plate, $100 \mu \mathrm{g}$ of proteins of DRG samples were used. ELISA was performed according to the manufacturer's protocol. The samples and the competition RvD1 tracer (RvD1 linked to acetylcholinesterase (AChE) were incubated overnight at $4{ }^{\circ} \mathrm{C}$. The signal in the ELISA plate was developed by Ellman's reagent, a substrate of AChE. The optical densities of samples were measured using an ELISA plate reader (Bio-Rad) at a wavelength of $420 \mathrm{~nm}$ and RvD1 levels were calculated using the standard curves. The standard curve was included in each experiment. The RvD1 values of the samples were in the linear range of the standard curve

anti-cancer and analgesic effects in mouse models of oral squamous cell carcinoma [64]. RvD2 reduced tumor size by inhibiting the proinflammatory cytokines IL- 6 and C-X-C motif chemokine 10 (CXCL10) and reduced tumor necrosis, a poor prognosticator of malignancies. RvD2 also produced transient analgesia in xenograft squamous cell carcinoma models, which was associated with decreased neutrophil infiltration and function [64].

\section{Protectin/Neuroprotectin D1}

Neuroprotectin D1 (NPD1) is derived from DHA, and the prefix "neuro" is indicative of the location of PD1 formation 
and actions in vivo. Within neural tissues, NPD1 exhibits potent neuroprotective actions in experimental brain damage, oxidative-stressed retinal pigment epithelial cells, and human brain cells exposed to $\beta$-amyloid peptides [50, 51, 56, 73]. NPD1 potently inhibits TRPV1 currents without affecting TRPA1 currents in dissociated mouse DRG neurons. This inhibition of TRPV1 is mediated by GPCRs, as the effects are blocked via application of pertussis toxin. Intrathecal administration is sufficient to block spinal long-term potentiation (LTP), a synaptic mechanism driving chronic pain [74]. NPD1 also reduces TNF-induced pain hypersensitivity [56].

In the CCI model of neuropathic pain, intrathecal administration of NPD1 also reduced mechanical allodynia at a dose that is 500 times lower than that of gabapentin [58]. Moreover, perisurgical application of NPD1 on the injured sciatic nerve around the ligature prevented nerve injury-induced mechanical allodynia and ongoing pain and neuroinflammation in the spinal cord in mice, demonstrating that NPD1 can act through both peripheral and central mechanisms. Intrathecal posttreatment of NPD1 2 weeks after CCI produced no apparent signs of analgesic tolerance, as seen in the reduction of mechanical allodynia [58]. Altogether, there is strong evidence to support the role of NPD1/PD1 treatment in blocking nerve injury-induced LTP, glial cell activation, and inflammatory responses, and these mechanisms drive chronic pain in the spinal cord [58].

\section{Maresins}

Another class of macrophage SPMs, termed maresins, is biosynthesized from DHA (Fig. 1). MaR1 potently inhibits polymorphonuclear neutrophil infiltration in murine peritonitis (ng/mouse range) and enhances human macrophage uptake of apoptotic neutrophils. Strikingly, MaR1 also promoted tissue regeneration by accelerating regeneration of head reappearance in planaria after postpharyngeal resection of the head. MaR1 dose-dependently inhibited TRPV1 currents in neurons, blocked capsaicin-induced inward currents, and reduced inflammatory pain in mice [75]. In mice, intrathecal postoperative treatment $(500 \mathrm{ng}) 2$ weeks after surgery with MaR1 reduced chemotherapy-induced neuropathic pain and bone fracture-induced postoperative pain [67, 75]. MaR1 also reduced CAG- and CFA-induced inflammatory pain and neutrophil and macrophage recruitment to the inflamed skin [76]. After spinal nerve ligation, intrathecal MaR1 administration inhibited neuropathic pain and spinal glial activation [77]. In a mouse model of rheumatoid arthritis, persistent pain was not associated with joint swelling, but instead, was associated with decreased concentrations of MaR1. Furthermore, systemic MaR1 administration caused sustained reversal of mechanical hypersensitivity and reduced inflammatory macrophage numbers in DRG [78]. Given that macrophages are critical contributors to chronic pain pathogenesis by forming bidirectional communication with nociceptors [79], it remains important to identify whether endogenous macrophagederived maresins contribute to chronic pain resolution.

\section{Lipoxins}

Lipoxins, such as lipoxin A4 (LXA4) and lipoxin B4 (LXA4), are products of arachidonic acid that require interaction with lipoxygenase for their biosynthesis [80]. Intravenous and intrathecal injection of LXA4 and LXB4 has been demonstrated to reduce CRG-induced inflammatory pain in rats [81]. Lipoxin receptor ALX was shown to be expressed in spinal cord astrocytes, and LXA4 prevented ATP-mediated phosphorylation of JNK (c-Jun N-terminal kinase), a MAPK family member, in primary astrocytes in vitro [81]. Notably, JNK activation in spinal cord astrocytes was implicated in inflammatory pain and neuropathic pain $[82,83]$. In the case of SCI, a severe neurological disorder with many disabling consequences, LXA4 has been shown to reduce neuroinflammation and neuropathic pain following SCI, including persistent neuropathic pain. LXA4 treatment produced significant attenuation of SCI-induced mechanical pain hypersensitivity, spinal microglial markers, and proinflammatory cytokines characteristic of SCI-induced glial activation. Important roles of LXA4 also include modulating microglial activation and TNF- $\alpha$ release through microglial ALX/FPR2 receptors, ultimately reducing neuropathic pain in rodents after spinal cord hemisection [60]. In a rat model of low back pain, intrathecal administration of LXA4 not only alleviated neuropathic pain but also inhibited the upregulation of proinflammatory cytokines TNF- $\alpha$ and IL- $1 \beta$ and increased the expression of antiinflammatory cytokines TGF- $\beta 1$ and IL-10 [84].

\section{Distinct Effects of DHA and DHA-Derived SPMs in Inflammatory Pain and Neuropathic Pain}

It is important to note that DHA and DHA-derived SPMs have distinct analgesic properties. For example, in contrast to DHA-derived SPMs, intrathecal DHA post-treatment had no effects on CCI-induced neuropathic pain or bone fractureinduced postoperative pain, even when administered at very high doses $(500 \mu \mathrm{g})[58,67]$. However, pretreatment of DHA in these conditions was effective [58,67]. A recent study also examined the analgesic impact of DHA and DHA-derived SPMs in a mouse model of postoperative pain induced by tibial bone fracture [67]. Intravenous perioperative treatment with DHA (500 $\mu \mathrm{g})$, RvD1 (500 ng), and MaR1 (500 ng), $10 \mathrm{~min}$ and $24 \mathrm{~h}$ after the surgery, delayed the development of fracture-induced postoperative pain. For direct comparison, intrathecal postoperative treatment $(500 \mathrm{ng})$ weeks after orthopedic surgery with NPD1, MaR1, RvD1, and RvD5 also effectively reduced mechanical and cold allodynia, yet posttreatment with DHA (500 $\mu$ g, i.t.) had no effects [67]. These 
findings suggest that DHA is only effective in pretreatment, whereas SPMs are effective in both pre- and post-treatment. Thus, distinct considerations exist for DHA and DHA-derived SPMs for treating chronic pain. It is also possible that the biosynthesis pathway for SPMs is impaired in chronic pain.

\section{SPMs Do Not Alter Physiological Pain}

Interestingly, despite the potent analgesic properties of resolvins in attenuating acute and chronic pain in pathological conditions, resolvins do not interfere with normal pain perception. Intraplantar, intrathecal, and systemic injection of resolvins were not observed to affect thermal or mechanical pain sensitivity in rats and mice under steady-state conditions [57]. In sharp contrast, classic opioid analgesics such as morphine dramatically decrease basal pain sensitivity. Under these basal conditions, disruption of physiological pain is not necessarily desirable or beneficial, as physiological pain has evolved as a protective response. Moreover, morphine can produce dose-dependent motor impairment. In contrast, intrathecal RvD2 reversed CFA-induced inflammatory pain without altering basal pain sensitivity or motor function [55]. RvD2 also abolished inflammation-induced sEPSC increases (frequency and amplitude), without affecting basal synaptic transmission [55]. Therefore, SPMs can serve to restore the homeostatic balance of pain without risk of swinging the balance too far in the opposite direction as opioid analgesics can.

\section{SPM Receptor Signaling Mechanisms in Pain}

SPMs exert their actions through GPCRs, and multiple SPM receptors have been identified to date (Fig. 1) [85]. The Dseries resolvins produce their beneficial actions via specific $G$ protein-coupled receptors, such as GPR32 for RvD1 and $\mathrm{RvD5}$, and GPR18 for RvD2 [86-88]. Interestingly, there is evidence that loss of SPM receptors may contribute to disease progression. For example, down-regulations of mRNA levels for Gpr 18 and Gpr32 were found in oral cancer cells [64]. In pain regulation, RvE1 inhibits inflammatory pain via ChemR23. Notably, ChemR23 is expressed in macrophages and microglia, as well as in TRPV1-expressing sensory neurons [52]. As another example, lipoxin A4 receptor ALXR is expressed on spinal astrocytes, and lipoxin A4 inhibited ATPinduced activation of MAPK (JNK and ERK) in cultured astrocytes [81]. Oehler et al. also showed that RvD1 and RvE1, through a peripheral interaction with the $\mu$-opioid receptor, contributing to the relief of inflammatory pain [89]. LRG6 was recently identified as a receptor for MaR 1 and implicated in phagocytosis [90], but its role in sensory neurons and pain remains to be investigated.

Recently, we demonstrated that NPD1 binds to GPR37, expressed in macrophages. NPD1 activation of GPR37 triggers increases in intracellular $\mathrm{Ca}^{2+}$, which regulates phagocytosis in macrophages [91]. Strikingly, this study demonstrated that NPD1 could trigger GPR37- and $\mathrm{Ca}^{2+}$-dependent macrophage phagocytosis of zymosan particles. Intraplantar injection of zymosan particles elicited inflammatory pain as well as local immune cell infiltration. The zymosan model offers unique advantages in studying inflammatory pain resolution mechanisms by enabling the examination of inflammation, phagocytosis, and inflammatory pain in tandem within the inflamed hindpaw skin. In this model, Gpr37 deficiency and macrophage depletion impaired the resolution of inflammatory pain by increasing the duration of pain. Conversely, adoptive transfer of WT but not Gpr37-deficient macrophage was sufficient to rescue resolution deficits in Gpr37-KO mice [91].

\section{SPMs and Sham Surgery}

In traditional preclinical studies involving surgical procedures, "sham surgery" (or placebo surgery) controls have been included to simulate the surgical operation, differing from the experimental condition only by the absence of the final intervention thought to be critically necessary (e.g., nerve injury, nerve crush, arthroscopy, etc.). Sham surgeries have also been used to test new surgical approaches in human patients. In this manner, the sham surgery control group simulates the nontreatment related surgical interventions in every nontreatment-related aspect such that investigators can reasonably attribute changes between sham and experimental groups to the intervention rather than the surgical procedures involved. Interestingly, sham surgery itself has been demonstrated to yield substantial benefits in a variety of different surgical conditions. In a systematic review comparing the baseline with sham- and active-treatment groups in more than 3500 patients receiving surgical interventions, it was estimated that sham surgery can account for approximately $65 \%$ of the overall improvements after surgery. Thus, only about onethird of the improvements were due to the surgical intervention being explored. Moreover, this effect was even more drastic when narrowed to include only patients with painful conditions, with sham surgery accounting for $78 \%$ of the overall improvements after surgery [92]. One frequently drawn conclusion from these studies is that these benefits are due to psychological factors influencing patient perception and their subsequent reaction to treatment (e.g. patients feel better because they expect they will). However, it is also possible that sham surgeries, which induce localized inflammatory responses, may also lead to improvements due to localized or systemic biochemical changes.

We recently explored whether sham surgery can lead to changes in RvD1, which has both anti-inflammatory and analgesic properties. We examined RvD1 levels using a recently developed ELISA kit (Cayman Chemical). RvD1 levels were measured in serum, spinal cord, and brain tissue samples of 
naïve mice, mice after sham surgery, and mice following bone fracture [67]. Samples were collected on day 5 after surgery, as DHA produced robust analgesic effects at this time point. Interestingly, compared to naïve animals and fracture surgery animals, sham surgery increased RvD1 levels in serum $(63.6 \mathrm{pg} / \mathrm{ml}$ in naïve and $273.6 \mathrm{pg} / \mathrm{ml}$ in sham, $P<0.05$ ) but not in brain or spinal cord samples, indicating that serum RvD1 level may be correlated with resolution of acute pain in sham animals. Our finding suggests that sham surgery may serve as a proresolution model. Thus, while links between inflammation and sustained pain are well-established, future studies exploring the protective effects of a mild localized inflammatory response on pain resolution are warranted.

\section{Biosynthetic Enzymes for SPMs}

The biosynthesis of SPMs has recently been reviewed [85], and several enzymes including COX-2 or cytochrome P450, and 5-lipoxygenase (5-LOX) are required for SPM biosynthesis from fish oil (DHA and EPA). Low-dose aspirin produces benefits in inflammation-associated chronic diseases, in part because aspirin initiates the biosynthetic pathway by acetylating COX-2 [52]. Notably, COX-2 is induced in spinal cord microglia and neurons after acute inflammation and plays an important role in inflammatory pain, although its role in persistent neuropathic pain is limited [93, 94]. Thus, biosynthesis for SPMs could be impaired in chronic pain due to transient expression of synthetic enzymes after injury. This may also explain the lack of efficacy in fish oil (e.g., DHA) for chronic pain, as there is an inability for SPM conversion in fish oil. It is of great interest to further investigate the temporal expression with detailed cellular localization of the biosynthetic enzymes of SPMs.

\section{Pharmacokinetics of SPMs}

As local acting lipid mediators, SPMs in general are metabolically unstable and can be rapidly inactivated and metabolized in vivo. Pharmacokinetics (PK) of SPMs could be estimated by the duration of pain relief. For example, intrathecal administration of RvE1 (10 $\mathrm{ng}$ ) reduced CFA-induced heat hyperalgesia for $<2 \mathrm{~h}$ [53]. Intrathecal administration of NPD1 (500 ng) reduced CCI-induced mechanical allodynia for $>3 \mathrm{~h}$, and furthermore, repeated injections of NPD1 did not produce analgesic tolerance [58], suggesting different PK of RvE1 and NPD1. Dehydrogenation of the hydroxyl group in tissue at carbon 18 position to form 18-oxo-RvE1 is the major initial metabolic route for RvE1, and accordingly, a modified form of RvE1 (19-pf-RvE1) is metabolically stable [95]. Notably, this stable form of RvE1 extended the antihyperalgesic effect of RvE1 from 2 to $6 \mathrm{~h}$ following intrathecal injection [53]. Furthermore, the anti-inflammatory and proresolving effects of AT-RvD1 have been prolonged by constructing novel nanoparticles that contain AT-RvD1 [96]. Hence, improving the PK of SMPs by developing more stable forms of SPMs or more stable delivery methods could prolong and enhance the analgesic and proresolving benefits of SPMs.

\section{Neuromodulation Controls Pain via Neural, Immune, and Glial Modulations}

Neuromodulation via electrical stimulation, such as spinal cord stimulation (SCS), deep brain stimulation (DBS), transcutaneous electrical nerve stimulation (TENS), vagus nerve stimulation (VNS), auricular stimulation, and DRG stimulation, as well as electroacupuncture (EA) has been used to provide pain relief in patients and animals $[15,97-101]$. Neuromodulation can also be achieved with transcranial magnetic stimulation (TMS) by inducing electrical currents in the brain [98], although TMS may also directly activate specific brain regions such as the suprachiasmatic nucleus [102]. Neuromodulation produces pain relief via activation of specific neural pathways [100, 102], suppression of nociceptive neuron activities (e.g., wide-dynamic neurons and projection neurons in the spinal cord $[103,104])$, and release of pain suppressing neurotransmitters and neuromodulators [101]. The gate control theory predicts that stimulation of large myelinated $\mathrm{A} \beta$ fibers inhibits $\mathrm{C}$-fiber-induced pain via a spinal cord inhibitory circuit [105]. Significant progress has been made in revealing the neurocircuits of the spinal cord "gate" in physiological and pathological conditions [26, 106-108]. SCS and EA could activate $A \beta$ fibers to suppress pain via gate control [103, 104, 109]. Interestingly, different EA frequency (e.g., 2, 15, $100 \mathrm{~Hz}$ ) may release different neuropeptides (e.g., enkephalin, endorphin, and dynorphin) for producing antinociception [101].

However, transient modulation of neuronal activity in the pain circuits during stimulation cannot account for long-term benefits of neuromodulation. Increasing evidence suggests that neuromodulation, such as VNS and SCS, can powerfully regulate inflammation and neuroinflammation [110, 111]. EA inhibits spinal IL- $1 \beta$ expression and attenuates bone cancer pain in rats [112]. In a rat model of neuropathic pain, SCS inhibits glial activation in the spinal cord [113]. EA and sciatic nerve activation regulate inflammation and immunity through vagus nerve activation and dopamine release [114]. In a rodent model of inflammatory muscle pain, manual acupuncture elicits antiinflammatory effects by stimulating the release of the antiinflammatory cytokine IL-10 from M2-like macrophages. In mice lacking IL-10, acupuncture became ineffective in reducing pain behaviors [115]. IL-10 was also implicated in EA analgesia in a mouse model of paw incisional pain [116].

VNS and auricular stimulation has been shown to alleviate multiple clinical pain conditions (Table 2), including fibromyalgia [117], pelvic pain [118], acute migraine [120-122], 
Table 2 Auricular vagus nerve stimulation reduces pain in multiple clinical conditions

\begin{tabular}{lll}
\hline Pain condition & Effects & References \\
\hline Fibromyalgia & Decrease widespread pain and tenderness & {$[117]$} \\
Pelvic pain & Decrease evoked pain intensity, temporal summation of pain and anxiety & {$[118]$} \\
Chronic migraine & Most patients reported pain relief ( $\geq 50 \%$ reduction in visual analog scale) & {$[119]$} \\
Acute migraine & $47 \%$ patients reported pain relief and 21\% patients reported pain free & {$[120]$} \\
Acute migraine & $38 \%$ patients had pain relief and 23\% patients had pain free & {$[121]$} \\
Acute migraine & $41 \%$ patients had pain relief and 30\% patients had pain free & {$[122]$} \\
$\begin{array}{l}\text { Episodic cluster } \\
\text { headache }\end{array}$ & Noninvasive VNS (48\%) is superior to sham (6\%) & {$[119]$} \\
In healthy & Increase mechanical and pressure pain threshold, decrease temporal & {$[123]$} \\
$\quad$ volunteers & summation of noxious tonic heat & \\
\hline
\end{tabular}

chronic migraine [119], and episodic cluster headache [123]. In 1984, Maixner and Randic demonstrated a role of antinociception of right vagal nerve trunk in animals [124]. The effect of transcutaneous VNS on pain perception was also investigated in healthy volunteers. A reduced sensitivity of mechanically evoked pain and an inhibition of temporal summation of noxious tonic heat in healthy volunteers point to the role of vagus nerve in regulation of basal pain perception [125]. Notably, EA causes neuronal activation in the nucleus of the solitary tract (NST) to mediate vagal responses [126, 127]. Tracy proposed the concept of "inflammatory reflex," suggesting there exists a neural circuit capable of providing information in real-time to the brain about the body's inflammatory status, therefore enabling a rapid neuron-mediated regulatory response through the vagus nerve [128].

Recent studies by Serhan and collaborators suggest that vagus nerve controls inflammation via the production of SPM [129]. Notably, vagotomy reduced local production of SPMs, such as RvD1 in exudates, thereby delaying resolution [130]. The human vagus produces SPMs (e.g., RvE1, NPD1/PD1, MaR1, RvD5, and LXA4), and electrical stimulation of the vagus nerve in vitro increased SPMs and decreased proinflammatory prostaglandins and leukotrienes [129, 131].

In a pilot study, we investigated whether auricular VNS via EA (aEA) would increase SPM production and inhibit neuropathic pain in a mouse model of CIPN, induced by paclitaxel (PTX) injections (Fig. 2a). Of importance, aEA significantly increased RvD1 level in DRG tissue $(P<0.01$, Fig. 2b, c). Systemic PTX treatment induced reduction in paw withdrawal threshold in both hind paws, indicating a development of bilateral mechanical allodynia. Notably, unilateral aEA, applied after PTX injections, effectively reversed mechanical allodynia on both paws $(P<0.001$, Fig. 2 d). Thus, auricular EA stimulation may alleviate neuropathic pain via producing SPMs (e.g., RvD1), which is supported by the finding that RvD1 was effective in attenuating mechanical allodynia even 2 weeks after PTX-induced CIPN in mice [61].

\section{Concluding Remarks and Future Directions}

At present, there are two ongoing and partially conflicting crises in the USA: the opioid epidemic and a crisis in chronic pain management. The rise in opioid prescriptions to treat a growing number of painful conditions was, at least in part, fueled by the superior efficacy of opioids in achieving pain relief compared to other pharmacotherapies. At the same time, this rise in opioid use led to a coincident rise in the number of Americans who experienced severe side effects, accidental overdose, and addiction, leading to the nationwide opioid epidemic [132]. This urgent crisis is being aggressively combated from many angles, including the rightful disuse of opioids for the majority of pain conditions. However, this contributes to a separate, but coevolving issue: a crisis in pain management. As the number of aging adults increases, the prevalence of chronic pain conditions and pain-related disability is also rising. Owing to the opioid epidemic and the subsequent disuse of opioids to treat the majority of chronic pain conditions, efficacious pharmacotherapies for pain management are lacking. Thus, there is a critical and urgent need to develop new strategies which provide safe, effective pain relief.

In this review, we have highlighted two promising, interrelated strategies for treating chronic pain: 1) SPMs, which exert direct analgesic actions while simultaneously promoting pain resolution, and 2) neuromodulation, which provides efficacious pain relief for many patients through neuronal cell-, glial cell-, and immune cell-mediated mechanisms, including the induction of SPMs. Compared to many traditional analgesics, one particular advantage offered by SPMs is their ability to potently control neuroinflammation, a critical underlying etiology which drives the maintenance of chronic pain. Thus, the combinatorial analgesic and anti-inflammatory properties of SPMs offers the potential to acutely suppress pain while concurrently promoting pain resolution, thereby offering both short- and long-term benefits. However, SPMs are unstable and expensive to produce, limiting their direct applications to patients. 
Dietary omega-3 fatty acids (e.g., DHA and EPA) are precursors of SPMs and were shown to alleviate inflammatory pain in patients [133], presumably via their own $G$ protein-coupled receptors (GPCRs) such as GRP120 [134]. However, the efficacy of these SPM precursors for pain relief is much lower compared to their metabolites. For example, the intrathecal dose required for either DHA and EPA to reduce CFA-induced heat hyperalgesia is $>1000$ times higher than that of RvE1. Thus, endogenous omega-3-derived resolvins may provide a missing link between dietary omega- 3 fatty acids and control of inflammation and pain ${ }^{79}$. In December 2019, the FDA approved an omega-3 fish oil medication, Vascepa (icosapent ethyl, EPA only), to reduce the risk of heart attack and stroke in patients at risk of cardiovascular disease, with $4 \mathrm{~g}$ daily taken to achieve therapeutic effects. High doses of omega-3 fatty acids may act to bolster biosynthesis of SPMs, which may yield unanticipated but welcomed beneficial effects. Thus, it will be interesting to follow unique cohorts of patients receiving these drugs and measure
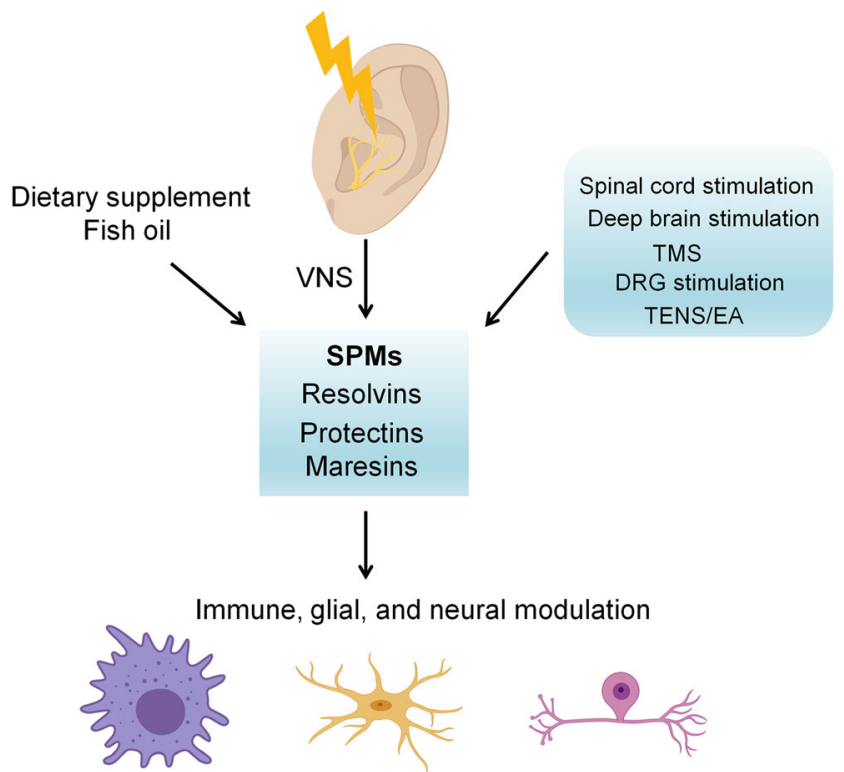

Immune cells

Glial cells

Neurons
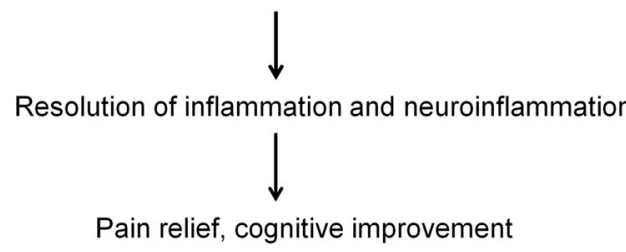

Fig. 3 Neuromodulation, such as VNS, spinal cord stimulation, deep brain stimulation, transcranial magnetic stimulation (TMS), transcutaneous nerve stimulation (TENS), and electroacupuncture (EA), promotes resolution of chronic pain via producing SPMs (e.g., RvD1). SPMs act on their receptors that are expressed on immune cells, glial cells, and neurons. Through SPM receptor signaling in different cell types, SPMs powerfully control inflammation and neuroinflammation, leading to pain relief and cognitive improvement after major surgeries, trauma, and infection. A combination of dietary supplements, such as fish oil and neuromodulation, may result in additive or synergistic beneficial effects by boosting the production of SPMs additional clinical parameters, such as pain in patients with chronic pain conditions as well as SPM profiles. Ramsdon and coworkers have already showed in a randomized trial that dietary omega-3 fatty acids may alleviate headache in patients by producing SPMs in a randomized trial $[135,136]$.

Even with many questions answered, key questions still remain: how do endogenous SPMs contribute to homeostasis, pain initiation, chronic pain maintenance, and pain resolution? These questions are challenging to answer but can be done so through several routes. First, given that host receptors for several SPMs have been identified (Fig. 1), knockout mice lacking these receptors can be utilized. However, this can be complicated by the fact that SPMs may act through multiple host receptors, and receptors may recognize multiple ligands. Thus, identifying host receptors and their signaling pathways that mediate the biological functions of different SPMs remains an important question. Additionally, given that SPMs are biosynthesized through well-characterized biochemical pathways from parent omega-3 fatty acids, studies investigating pain pathogenesis in preclinical models with specific dietary restrictions (e.g., arachidonic acid deficiency, DHA deficiency) offer another route of interrogation. It will also be of great interest to study a synergistic effect between healthy diet and neuromodulation for the resolution of pain through regulation of inflammation and neuroinflammation (Fig. 3).

Acknowledgments The work was supported by Duke University Research Funds. Christopher Donnelly was supported by Bonica Fellowship from the International Association for the Study of Pain (IASP).

Required Author Forms Disclosure forms provided by the authors are available with the online version of this article.

\section{Compliance with Ethical Standards}

Conflict of Interest The authors declare that they have no conflict of interest.

\section{References}

1. Woolf, C.J. and Q. Ma, Nociceptors-noxious stimulus detectors. Neuron, 2007. 55(3): p. 353-364.

2. Ji, R.R., Z.Z. Xu, and Y.J. Gao, Emerging targets in neuroinflammation-driven chronic pain. Nat.Rev.Drug Discov., 2014. 13(7): p. 533-548.

3. Basbaum, A.I., et al., Cellular and molecular mechanisms of pain. Cell, 2009. 139(2): p. 267-284.

4. Park, C.K., et al., Extracellular MicroRNAs Activate Nociceptor Neurons to Elicit Pain via TLR7 and TRPA1. Neuron, 2014. 82(1): p. 47-54.

5. Julius, D. and A.I. Basbaum, Molecular mechanisms of nociception. Nature, 2001. 413(6852): p. 203-210.

6. Gold, M.S., J.D. Levine, and A.M. Correa, Modulation of TTX-R $I N a$ by PKC and PKA and their role in PGE2-induced 
sensitization of rat sensory neurons in vitro. J.Neurosci., 1998. 18(24): p. 10345-10355.

7. Sisignano, M., et al., 5,6-EET is released upon neuronal activity and induces mechanical pain hypersensitivity via TRPA1 on central afferent terminals. J Neurosci, 2012. 32(18): p. 6364-72.

8. White, F.A., S.K. Bhangoo, and R.J. Miller, Chemokines: integrators of pain and inflammation. Nat.Rev.Drug Discov., 2005. 4(10): p. 834-844.

9. Hokfelt, T., X. Zhang, and Z. Wiesenfeld-Hallin, Messenger plasticity in primary sensory neurons following axotomy and its functional implications. Trends Neurosci., 1994. 17(1): p. 22-30.

10. Lai NY, et al., Gut-Innervating Nociceptor Neurons Regulate Peyer's Patch Microfold Cells and SFB Levels to Mediate Salmonella Host Defense. Cell, 2019.

11. Baral, P., S. Udit, and I.M. Chiu, Pain and immunity: implications for host defence. Nat Rev Immunol, 2019. 19(7): p. 433-447.

12. Gold, M.S. and G.F. Gebhart, Nociceptor sensitization in pain pathogenesis. Nat.Med., 2010. 16(11): p. 1248-1257.

13. Hucho, T. and J.D. Levine, Signaling pathways in sensitization: toward a nociceptor cell biology. Neuron, 2007. 55(3): p. 365376.

14. Woolf, C.J., Central sensitization: implications for the diagnosis and treatment of pain. Pain, 2011. 152(3 Suppl): p. S2-15.

15. Ji, R.R., et al., Neuroinflammation and Central Sensitization in Chronic and Widespread Pain. Anesthesiology, 2018.

16. Usoskin, D., et al., Unbiased classification of sensory neuron types by large-scale single-cell RNA sequencing. Nat.Neurosci, 2015. 18(1): p. 145-153.

17. Zheng, Y., et al., Deep Sequencing of Somatosensory Neurons Reveals Molecular Determinants of Intrinsic Physiological Properties. Neuron, 2019.

18. Ji, R.R., et al., $p 38$ MAPK activation by NGF in primary sensory neurons after inflammation increases TRPV1 levels and maintains heat hyperalgesia. Neuron, 2002. 36(1): p. 57-68.

19. Obata, K., et al., TRPA1 induced in sensory neurons contributes to cold hyperalgesia after inflammation and nerve injury. J.Clin.Invest, 2005. 115(9): p. 2393-2401.

20. Binshtok, A.M., et al., Nociceptors are interleukin-1beta sensors. J.Neurosci., 2008. 28(52): p. 14062-14073.

21. Jin, X. and R.W. Gereau, Acute p38-mediated modulation of tetrodotoxin-resistant sodium channels in mouse sensory neurons by tumor necrosis factor-alpha. J.Neurosci, 2006. 26(1): p. 246255.

22. Latremoliere, A. and C.J. Woolf, Central sensitization: a generator of pain hypersensitivity by central neural plasticity. J.Pain, 2009. 10(9): p. 895-926.

23. Ji, R.R., et al., Central sensitization and LTP: do pain and memory share similar mechanisms? Trends Neurosci., 2003. 26(12): p. 696-705.

24. Moore, K.A., et al., Partial peripheral nerve injury promotes a selective loss of GABAergic inhibition in the superficial dorsal horn of the spinal cord. J.Neurosci., 2002. 22(15): p. 6724-6731.

25. Coull, J.A., et al., Trans-synaptic shift in anion gradient in spinal lamina I neurons as a mechanism of neuropathic pain. Nature, 2003. 424(6951): p. 938-942.

26. Lu, Y., et al., A feed-forward spinal cord glycinergic neural circuit gates mechanical allodynia. J Clin.Invest, 2013. 123(9): p. 40504062.

27. Yang, Q., et al., Persistent pain after spinal cord injury is maintained by primary afferent activity. J Neurosci, 2014. 34(32): p. 10765-9.

28. Ellis, A. and D.L. Bennett, Neuroinflammation and the generation of neuropathic pain. Br.J Anaesth, 2013. 111(1): p. 26-37.

29. Ji, R.R., T. Berta, and M. Nedergaard, Glia and pain: Is chronic pain a gliopathy? Pain, 2013.
30. Clark, A.K., et al., P2X7-dependent release of interleukin-1beta and nociception in the spinal cord following lipopolysaccharide. J.Neurosci., 2010. 30(2): p. 573-582.

31. Inoue, K. and M. Tsuda, Microglia in neuropathic pain: cellular and molecular mechanisms and therapeutic potential. Nat Rev Neurosci, 2018. 19(3): p. 138-152.

32. Milligan, E.D., et al., Spinal glia and proinflammatory cytokines mediate mirror-image neuropathic pain in rats. J.Neurosci., 2003. 23(3): p. 1026-1040

33. Coull, J.A., et al., BDNF from microglia causes the shift in neuronal anion gradient underlying neuropathic pain. Nature, 2005. 438(7070): p. 1017-1021.

34. Kawasaki, Y., et al., Cytokine mechanisms of central sensitization: distinct and overlapping role of interleukin-1beta, interleukin-6, and tumor necrosis factor-alpha in regulating synaptic and neuronal activity in the superficial spinal cord. J.Neurosci, 2008. 28(20): p. 5189-5194.

35. Luo, H., et al., Interleukin-17 Regulates Neuron-Glial Communications, Synaptic Transmission, and Neuropathic Pain after Chemotherapy. Cell Rep, 2019. 29(8): p. 2384-2397 e5.

36. Chiu, I.M., C.A. von Hehn, and C.J. Woolf, Neurogenic inflammation and the peripheral nervous system in host defense and immunopathology. Nat.Neurosci, 2012. 15(8): p. 1063-1067.

37. Matsuda, M., Y. Huh, and R.R. Ji, Roles of inflammation, neurogenic inflammation, and neuroinflammation in pain. $\mathrm{J}$ Anesth, 2019. 33(1): p. 131-139.

38. Wei, T., et al., Post-junctional facilitation of Substance P signaling in a tibia fracture rat model of complex regional pain syndrome type I. Pain, 2009. 144(3): p. 278-86.

39. Gilroy, D.W., et al., Inducible cyclooxygenase may have antiinflammatory properties. Nat.Med., 1999. 5(6): p. 698-701.

40. Fullerton, J.N. and D.W. Gilroy, Resolution of inflammation: a new therapeutic frontier. Nat Rev Drug Discov, 2016. 15(8): $\mathrm{p}$. 551-67.

41. Buckley, C.D., D.W. Gilroy, and C.N. Serhan, Proresolving lipid mediators and mechanisms in the resolution of acute inflammation. Immunity, 2014. 40(3): p. 315-27.

42. Bannenberg, G. and C.N. Serhan, Specialized pro-resolving lipid mediators in the inflammatory response: An update. Biochim.Biophys.Acta, 2010. 1801(12): p. 1260-1273.

43. Serhan, C.N., Pro-resolving lipid mediators are leads for resolution physiology. Nature, 2014. 510(7503): p. 92-101.

44. Norling, L.V., et al., Proresolving and cartilage-protective actions of resolvin D1 in inflammatory arthritis. JCI Insight, 2016. 1(5): p. e85922.

45. Norris PC, et al., Resolvin D3 multi-level proresolving actions are host protective during infection. Prostaglandins Leukot Essent Fatty Acids, 2016.

46. Sulciner, M.L., et al., Resolvins suppress tumor growth and enhance cancer therapy. J Exp Med, 2018. 215(1): p. 115-140.

47. Duffield, J.S., et al., Resolvin D series and protectin D1 mitigate acute kidney injury. J.Immunol., 2006. 177(9): p. 5902-5911.

48. Spite, M., et al., Resolvin D2 is a potent regulator of leukocytes and controls microbial sepsis. Nature, 2009. 461(7268): p. 1287 1291.

49. Arita, M., et al., Stereochemical assignment, antiinflammatory properties, and receptor for the omega-3 lipid mediator resolvin E1. J.Exp.Med., 2005. 201(5): p. 713-722.

50. Lukiw, W.J. and N.G. Bazan, Inflammatory, apoptotic, and survival gene signaling in Alzheimer's disease. A review on the bioactivity of neuroprotectin D1 and apoptosis. Mol.Neurobiol., 2010. 42(1): p. 10-16.

51. Lukiw, W.J., et al., A role for docosahexaenoic acid-derived neuroprotectin D1 in neural cell survival and Alzheimer disease. J.Clin.Invest, 2005. 115(10): p. 2774-2783. 
52. Ji, R.R., et al., Emerging roles of resolvins in the resolution of inflammation and pain. Trends Neurosci., 2011.

53. $\mathrm{Xu}, \mathrm{Z} . \mathrm{Z}$., et al., Resolvins RvE1 and RvD1 attenuate inflammatory pain via central and peripheral actions. Nat.Med., 2010. 16(5): p. 592-7, 1p.

54. Bang, S., et al., Resolvin D1 attenuates activation of sensory transient receptor potential channels leading to multiple antinociception. Br.J.Pharmacol., 2010. 161(3): p. 707-720.

55. Park, C.K., et al., Resolvin d2 is a potent endogenous inhibitor for transient receptor potential subtype v1/al, inflammatory pain, and spinal cord synaptic plasticity in mice: distinct roles of resolvin $d 1$, $d 2$, and e1. J.Neurosci., 2011. 31(50): p. 1843318438.

56. Park, C.K., et al., Resolving TRPV1- and TNF- $\alpha$-mediated spinal cord synaptic plasticity and inflammatory pain with neuroprotectin D1. J Neurosci, 2011. 31(42): p. 15072-15085.

57. Xu, Z.Z. and R.R. Ji, Resolvins are potent analgesics for arthritic pain. Br.J.Pharmacol., 2011. 164(2): p. 274-277.

58. Xu, Z.Z., et al., Neuroprotectin/protectin D1 protects against neuropathic pain in mice after nerve trauma. Ann Neurol, 2013. 74(3): p. 490-5.

59. Xu, Z.Z., T. Berta, and R.R. Ji, Resolvin E1 inhibits neuropathic pain and spinal cord microglial activation following peripheral nerve injury. J Neuroimmune Pharmacol., 2013. 8(1): p. 37-41.

60. Martini, A.C., et al., Lipoxin A4 inhibits microglial activation and reduces neuroinflammation and neuropathic pain after spinal cord hemisection. J Neuroinflammation, 2016. 13(1): p. 75.

61. Luo, X., et a1., Resolvin D5 Inhibits Neuropathic and Inflammatory Pain in Male But Not Female Mice: Distinct Actions of D-Series Resolvins in Chemotherapy-Induced Peripheral Neuropathy. Front Pharmacol, 2019. 10: p. 745.

62. Huang, L., et al., Enduring prevention and transient reduction of postoperative pain by intrathecal resolvin D1. Pain, 2011. 152(3): p. $557-565$.

63. Wang JC and Strichartz GR, Prevention of Chronic PostThoracotomy Pain in Rats By Intrathecal Resolvin D1 and D2: Effectiveness of Perioperative and Delayed Drug Delivery. J Pain, 2017.

64. Ye, Y., et al., Anti-cancer and analgesic effects of resolvin D2 in oral squamous cell carcinoma. Neuropharmacology, 2018. 139: p. 182-193.

65. Patapoutian, A., S. Tate, and C.J. Woolf, Transient receptor potential channels: targeting pain at the source. Nat.Rev.Drug Discov., 2009. 8(1): p. 55-68.

66. Dixon, W.J., Efficient analysis of experimental observations. Annu.Rev.Pharmacol.Toxicol., 1980. 20: p. 441-462.

67. Zhang, L., et al., Distinct Analgesic Actions of DHA and DHADerived Specialized Pro-Resolving Mediators on Post-operative Pain After Bone Fracture in Mice. Front Pharmacol, 2018. 9: p. 412

68. Flatters, S.J., Characterization of a model of persistent postoperative pain evoked by skin/muscle incision and retraction (SMIR). Pain, 2008. 135(1-2): p. 119-130.

69. Li, Y., et al., Toll-like receptor 4 signaling contributes to Paclitaxel-induced peripheral neuropathy. J Pain, 2014. 15(7): p. 712-725.

70. Flatters, S.J. and G.J. Bennett, Ethosuximide reverses paclitaxeland vincristine-induced painful peripheral neuropathy. Pain, 2004. 109(1-2): p. 150-161.

71. Sisignano, M., et al., Mechanism-based treatment for chemotherapy-induced peripheral neuropathic pain. Nat Rev Neurol, 2014. 10(12): p. 694-707.
72. Cavaletti, G. and P. Marmiroli, Chemotherapy-induced peripheral neurotoxicity. Nat.Rev.Neurol., 2010. 6(12): p. 657-666.

73. Palacios-Pelaez, R., W.J. Lukiw, and N.G. Bazan, Omega-3 essential fatty acids modulate initiation and progression of neurodegenerative disease. Mol.Neurobiol., 2010. 41(2-3): p. 367-374.

74. Ruscheweyh, R., et al., Long-term potentiation in spinal nociceptive pathways as a novel target for pain therapy. Mol.Pain, 2011. 7: p. 20.

75. Serhan, C.N., et al., Macrophage proresolving mediator maresin 1 stimulates tissue regeneration and controls pain. FASEB J., 2012. 26(4): p. 1755-1765.

76. Fattori, V., et al., The specialised pro-resolving lipid mediator maresin 1 reduces inflammatory pain with a long-lasting analgesic effect. Br J Pharmacol, 2019. 176(11): p. 1728-1744.

77. Gao, J., et al., Pro-resolving mediator maresin 1 ameliorates pain hypersensitivity in a rat spinal nerve ligation model of neuropathic pain. J Pain Res, 2018. 11: p. 1511-1519.

78. Allen, B.L., et al., Imbalance of pro-resolving lipid mediators in persistent allodynia dissociated from signs of clinical arthritis. Pain, 2020

79. Chen, O., C.R. Donnelly, and R.R. Ji, Regulation of pain by neuro-immune interactions between macrophages and nociceptor sensory neurons. Curr Opin Neurobiol, 2019. 62: p. 17-25.

80. Serhan, C.N., M. Hamberg, and B. Samuelsson, Lipoxins: novel series of biologically active compounds formed from arachidonic acid in human leukocytes. Proc Natl Acad Sci U S A, 1984. 81(17): p. 5335-9.

81. Svensson, C.I., M. Zattoni, and C.N. Serhan, Lipoxins and aspirin-triggered lipoxin inhibit inflammatory pain processing. J.Exp.Med., 2007. 204(2): p. 245-252.

82. Zhuang, Z.Y., et al., A peptide c-Jun N-terminal kinase (JNK) inhibitor blocks mechanical allodynia after spinal nerve ligation: respective roles of JNK activation in primary sensory neurons and spinal astrocytes for neuropathic pain development and maintenance. J.Neurosci, 2006. 26(13): p. 3551-3560.

83. Gao, Y.J., et al., The c-Jun N-terminal kinase 1 (JNK1) in spinal astrocytes is required for the maintenance of bilateral mechanical allodynia under a persistent inflammatory pain condition. Pain, 2010. 148(2): p. 309-319.

84. Miao, G.S., et al., Lipoxin A4 attenuates radicular pain possibly by inhibiting spinal ERK, JNK and NF-kappaB/p65 and cytokine signals, but not $p 38$, in a rat model of non-compressive lumbar disc herniation. Neuroscience, 2015. 300: p. 10-8.

85. Chen, G., et al., Microglia in Pain: Detrimental and Protective Roles in Pathogenesis and Resolution of Pain. Neuron, 2018. 100(6): p. 1292-1311.

86. Chiang, N., et al., Infection regulates pro-resolving mediators that lower antibiotic requirements. Nature, 2012. 484(7395): p. 524528.

87. Chiang, N., et al., Identification of resolvin D2 receptor mediating resolution of infections and organ protection. J Exp Med, 2015. 212(8): p. 1203-17.

88. Krishnamoorthy, S., et al., Resolvin D1 binds human phagocytes with evidence for proresolving receptors. Proc.Natl.Acad.Sci.U.S.A, 2010. 107(4): p. 1660-1665.

89. Oehler, B., et al., Peripheral Interaction of Resolvin D1 and E1 with Opioid Receptor Antagonists for Antinociception in Inflammatory Pain in Rats. Front Mol Neurosci, 2017. 10: p. 242.

90. Chiang, N., et al., Maresin 1 activates LGR6 receptor promoting phagocyte immunoresolvent functions. J Clin Invest, 2019. 129(12): p. 5294-5311. 
91. Bang, S., et al., GPR37 regulates macrophage phagocytosis and resolution of inflammatory pain. J Clin Invest, 2018. 128(8): p. 3568-3582.

92. Jonas, W.B., et al., To what extent are surgery and invasive procedures effective beyond a placebo response? A systematic review with meta-analysis of randomised, sham controlled trials. BMJ Open, 2015. 5(12):p. e009655.

93. Samad, T.A., et al., Interleukin-1beta-mediated induction of Cox-2 in the CNS contributes to inflammatory pain hypersensitivity. Nature, 2001. 410(6827): p. 471-475.

94. Svensson, C.I., et al., Activation of $p 38$ mitogen-activated protein kinase in spinal microglia is a critical link in inflammationinduced spinal pain processing. J.Neurochem., 2003. 86(6): p. 1534-1544.

95. Arita, M., et al., Metabolic inactivation of resolvin E1 and stabilization of its anti-inflammatory actions. J.Biol.Chem., 2006. 281(32): p. 22847-22854.

96. Norling, L.V., et al., Cutting edge: humanized nano-proresolving medicines mimic inflammation-resolution and enhance wound healing. J.Immunol., 2011. 186(10): p. 5543-5547.

97. Guan, Y., et al., Spinal cord stimulation-induced analgesia: electrical stimulation of dorsal column and dorsal roots attenuates dorsal horn neuronal excitability in neuropathic rats. Anesthesiology, 2010. 113(6): p. 1392-405.

98. Moreno-Duarte, I., et al., Targeted therapies using electrical and magnetic neural stimulation for the treatment of chronic pain in spinal cord injury. Neuroimage, 2014. 85 Pt 3: p. 1003-13.

99. Shamji, M.F., C. De Vos, and A. Sharan, The Advancing Role of Neuromodulation for the Management of Chronic TreatmentRefractory Pain. Neurosurgery, 2017. 80(3S): p. S108-S113.

100. Pawela, C.P., J.M. Kramer, and Q.H. Hogan, Dorsal root ganglion stimulation attenuates the BOLD signal response to noxious sensory input in specific brain regions: Insights into a possible mechanism for analgesia. Neuroimage, 2017. 147: p. 10-18.

101. Han, J.S., Acupuncture: neuropeptide release produced by electrical stimulation of different frequencies. Trends Neurosci, 2003. 26(1): p. 17-22.

102. Ji, R.R., et al., Repetitive transcranial magnetic stimulation activates specific regions in rat brain. Proc.Natl.Acad.Sci.U.S.A, 1998. 95(26): p. 15635-15640.

103. Yang, F., et al., Effects of Combined Electrical Stimulation of the Dorsal Column and Dorsal Roots on Wide-Dynamic-Range Neuronal Activity in Nerve-Injured Rats. Neuromodulation, 2015. 18(7): p. 592-7; discussion 598.

104. Zhang, T.C., et al., Spinal sensory projection neuron responses to spinal cord stimulation are mediated by circuits beyond gate control. J Neurophysiol, 2015. 114(1): p. 284-300.

105. Melzack, R. and P.D. Wall, Pain mechanisms: a new theory. Science, 1965. 150(3699): p. 971-979.

106. Duan, B., L. Cheng, and Q. Ma, Spinal Circuits Transmitting Mechanical Pain and Itch. Neurosci Bull, 2018. 34(1): p. 186193.

107. Braz, J., et al., Transmitting Pain and Itch Messages: A Contemporary View of the Spinal Cord Circuits that Generate Gate Control. Neuron, 2014. 82(3): p. 522-536.

108. Peirs, C. and R.P. Seal, Neural circuits for pain: Recent advances and current views. Science, 2016. 354(6312): p. 578-584.

109. Huang, Q., et al., Spinal Cord Stimulation for Pain Treatment After Spinal Cord Injury. Neurosci Bull, 2019. 35(3): p. 527-539.

110. Borovikova, L.V., et al., Vagus nerve stimulation attenuates the systemic inflammatory response to endotoxin. Nature, 2000. 405(6785): p. 458-62.
111. Steinberg, B.E., et al., Neural Control of Inflammation: Implications for Perioperative and Critical Care. Anesthesiology, 2016. 124(5): p. 1174-89.

112. Zhang, R.X., et al., Electroacupuncture attenuates bone cancer pain and inhibits spinal interleukin-1 beta expression in a rat model. Anesth Analg, 2007. 105(5): p. 1482-8, table of contents.

113. Sato, K.L., et al., Spinal cord stimulation reduces mechanical hyperalgesia and glial cell activation in animals with neuropathic pain. Anesth Analg, 2014. 118(2): p. 464-72.

114. Torres-Rosas, R., et al., Dopamine mediates vagal modulation of the immune system by electroacupuncture. Nat Med, 2014. 20(3): p. 291-5.

115. da Silva, M.D., et al., IL-10 cytokine released from M2 macrophages is crucial for analgesic and anti-inflammatory effects of acupuncture in a model of inflammatory muscle pain. Mol Neurobiol, 2015. 51(1): p. 19-31.

116. Dai, W.J., et al., Involvement of Interleukin-10 in Analgesia of Electroacupuncture on Incision Pain. Evid Based Complement Alternat Med, 2019. 2019: p. 8413576.

117. Lange, G., et al., Safety and efficacy of vagus nerve stimulation in fibromyalgia: a phase I/II proof of concept trial. Pain Med, 2011. 12(9): p. 1406-13.

118. Napadow, V., et al., Evoked pain analgesia in chronic pelvic pain patients using respiratory-gated auricular vagal afferent nerve stimulation. Pain Med, 2012. 13(6): p. 777-89.

119. Barbanti, P., et al., Non-invasive vagus nerve stimulation for acute treatment of high-frequency and chronic migraine: an open-label study. J Headache Pain, 2015. 16: p. 61.

120. Goadsby, P.J., et al., Effect of noninvasive vagus nerve stimulation on acute migraine: an open-label pilot study. Cephalalgia, 2014. 34(12): p. 986-93.

121. Martelletti, P., et al., Consistent effects of non-invasive vagus nerve stimulation (nVNS) for the acute treatment of migraine: additional findings from the randomized, sham-controlled. double-blind PRESTO trial. J Headache Pain, 2018. 19(1): p. 101.

122. Tassorelli, C., et al., Noninvasive vagus nerve stimulation as acute therapy for migraine: The randomized PRESTO study. Neurology, 2018. 91(4): p. e364-e373.

123. Goadsby, P.J., et al., Non-invasive vagus nerve stimulation for the acute treatment of episodic and chronic cluster headache: A randomized, double-blind, sham-controlled ACT2 study. Cephalalgia, 2018. 38(5): p. 959-969.

124. Maixner, W. and A. Randich, Role of the right vagal nerve trunk in antinociception. Brain Res, 1984. 298(2): p. 374-7.

125. Busch, $\mathrm{V}$., et al., The effect of transcutaneous vagus nerve stimulation on pain perception-an experimental study. Brain Stimul, 2013. 6(2): p. 202-9.

126. Fang, J.F., et al., Effect of Electroacupuncture on the NTS is modulated primarily by acupuncture point selection and stimulation frequency in normal rats. BMC Complement Altern Med, 2017. 17(1): p. 182.

127. Lim, H.D., et al., Anti-Inflammatory Effects of Acupuncture Stimulation via the Vagus Nerve. PLoS One, 2016. 11(3): p. e0151882.

128. Tracey, K.J., The inflammatory reflex. Nature, 2002. 420(6917): p. 853-9.

129. Serhan, C.N., X. de la Rosa, and C. Jouvene, Novel mediators and mechanisms in the resolution of infectious inflammation: evidence for vagus regulation. J Intern Med, 2019. 286(3): p. 240-258.

130. Mirakaj, V., et al., Vagus nerve controls resolution and proresolving mediators of inflammation. J Exp Med, 2014. 211(6): p. $1037-48$. 
131. Serhan, C.N., X. de la Rosa, and C.C. Jouvene, Cutting Edge: Human Vagus Produces Specialized Proresolving Mediators of Inflammation with Electrical Stimulation Reducing Proinflammatory Eicosanoids. J Immunol, 2018. 201(11): p. 3161-3165.

132. Volkow, N.D. and F.S. Collins, The Role of Science in Addressing the Opioid Crisis. N Engl J Med, 2017. 377(4): p. 391-394.

133. Goldberg, R.J. and J. Katz, A meta-analysis of the analgesic effects of omega-3 polyunsaturated fatty acid supplementation for inflammatory joint pain. Pain, 2007. 129(1-2): p. 210-223.

134. Oh, D.Y., et al., GPR120 is an omega-3 fatty acid receptor mediating potent anti-inflammatory and insulin-sensitizing effects. Cell, 2010. 142(5): p. 687-698.
135. Ramsden, C.E., et al., Targeted alterations in dietary $n-3$ and n-6 fatty acids improve life functioning and reduce psychological distress among patients with chronic headache: a secondary analysis of a randomized trial. Pain, 2015. 156(4): p. 587-96.

136. Ramsden, C.E., et al., Targeted alteration of dietary n-3 and n-6 fatty acids for the treatment of chronic headaches: a randomized trial. Pain, 2013. 154(11): p. 2441-2451.

Publisher's Note Springer Nature remains neutral with regard to jurisdictional claims in published maps and institutional affiliations. 